\title{
Comparison of Four Distinct Idiotypes of Monoclonal Antibodies against Hen's Egg Ovomucoid
}

\author{
Atsushi Enomoto, Yoshikazu Aoki, Jun-ichi Kurisaki, \\ Shuichi Kaminogawa and Kunio Yamauchi \\ Department of Agricultural Chemistry, The University of Tokyo, \\ Bunkyo-ku, Tokyo, 113, Japan
}

Received June 24, 1987

\begin{abstract}
Two monoclonal antibodies ( $m A b 41 B 3$ and $m A b 51 B 3$ ), directed against hen's egg ovomucoid $(\mathrm{OM})$ and different from those we previously reported ( $m A b 23 E 5$ and $m A b 32 A 8$ ), were prepared and purified. A competitive radioimmunoassay using ${ }^{125} \mathrm{I}$-labeled OM showed that both $m A b 41 B 3$ and $m A b 51 B 3$ could bind all three homologous domains (domains I (DI), II (DII) and III (DIII)) of $\mathrm{OM}$ and that they reacted most efficiently with DIII. To analyze the paratope specificities of these four $\mathrm{mAbs}$, a sandwich assay and a competitive radioimmunoassay were done. Only the pair $m A b 41 B 3$ and $m A b 51 B 3$ could not simultaneously bind the OM or domains in the sandwich assay. Only $m A b 41 B 3$ inhibited the binding of $m A b 51 B 3$ to antigens and vice versa in the competitive radioimmunoassay. These results suggest that $m A b 41 B 3$ and $m A b 51 B 3$ recognized a closely related site distinct from the epitopes for $m A b 23 E 5$ or $m A b 32 A 8$. These mAbs may be useful for general studies on epitopes of protein antigens as well as for analyses of the antigenic determinants of OM.
\end{abstract}

Although hen's eggs are considered a good food, they contain proteins that cause allergic reactions in some humans, particularly in children. ${ }^{1)}$ Ovomucoid (OM), a glycoprotein in egg white, is a major allergen of eggs. ${ }^{2)}$ The primary structure of OM is known ${ }^{3)}$; it consists of three homologous domains (domain I (DI), domain II (DII), and domain III (DIII)) that have heterogenous sugar chains. ${ }^{4)}$

To study the antigenic sites of OM, we had prepared, purified, and characterized two monoclonal antibodies ( $m A b 23 E 5$ and $m A b$ $32 A 8$ ) against $\mathrm{OM} .^{5)}$ Both $\mathrm{mAbs}$ could bind to every domain of OM, but their affinity was not equal. $M A b 23 E 5$ bound more efficiently to fragments containing DIII than to fragments without DIII. $M A b 32 A 8$ reacted most efficiently with DIII-2 (with carbohydrates) and least efficiently with DIII-1 (free from carbohydrates) in all the domains. These results suggested that $m A b 23 E 5$ mainly recognized a region on the polypeptide chain of DIII and that $m A b 32 A 8$ might have recognized a region located on the carbohydrate moiety of
OM.

However, these two mAbs cannot by themselves reveal all the antigenic structures of OM. For this paper, we have prepared, purified, and characterized two new mAbs ( $m A b 41 B 3$ and $m A b 51 B 3$ ) distinct from the previous two. To test whether the mAbs recognized different epitopes on OM, a sandwich assay and solid-phase competitive radioimmunoassay (RIA) were used. These assays could determine whether the two mAbs could simultaneously bind to one ovomucoid molecule (i.e., whether they recognized the distinct epitopes) or not (i.e., whether they could recognize the same epitope or two close sites). We found that $m A b 41 B 3$ and $m A b 51 B 3$ could bind to closely related sites (containing the same site) different from the epitopes for $m A b$ $23 E 5$ or $m A b 32 A 8$.

In this paper, we describe the preparation of the new mAbs specific for OM, their reactions with ovomucoid and its domains, and their paratope specificity. 


\section{MATERIALS AND METHODS}

Mice. Mice (BALB/c) were purchased from Charles River Japan Inc.

Antigens. Eggs of White Leghorn hens were obtained from the National Institute of the Animal Industry, Ibaraki, Japan. OM was prepared from the eggs by the method of Fredericq and Deutsch, ${ }^{6)}$ and purified by DEAE-Sephacel ion-exchange chromatography. Domains (DI, II, III-1, III-2, I-II, II-III) were prepared by combining enzymic digestion (using Staphylococcus aureus V.8 protease from Miles Laboratories) ${ }^{7)}$ and chemical cleavage of the peptide bonds (using cyanogen bromide),${ }^{8)}$ and were then purified by gel filtration on Bio-Gel P-30 or affinity chromatography on trypsinSepharose 4B. ${ }^{9)}$ Reduced and carboxymethylated OM (RCM-OM) was obtained by the method of Konigsberg. ${ }^{10)}$

Immunization. Four- to six-week-old female BALB/c mice were immunized intraperitoneally with $100 \mu \mathrm{g}$ of purified OM in complete Freund's adjuvant (CFA). They were boosted with a similar dose in incomplete Freund's adjuvant (IFA) after 2 weeks, and then intravenously injected without any adjuvant 3 days before cell fusion.

Cell fusion. Cells were fused by the general method of Köhler and Milstein. ${ }^{11)}$ Spleen cells of immunized BALB/c mice were harvested and hybridized with X63-Ag.8-6.5.3 mouse myeloma cells ( $1 / 10$ of the number of spleen cells), using $45 \%$ polyethyleneglycol (M.W. 3350 ) at $37^{\circ} \mathrm{C}$. The cells were seeded in 24-well culture plates $\left(1.0 \times 10^{6}\right.$ spleen cells/well) and were fed 14 days after hybridization in HAT medium for HAT selection. The supernatants from the growing culture were tested for antibody activity by an enzyme-linked immunosorbent assay (ELISA) ${ }^{12)}$ with OM as the coated antigen. Hybridomas producing anti-OM antibodies were cloned twice by limiting dilution with normal thymocytes as feeders. The cloned anti-OM antibody-producing lines were grown by tissue culture, and some were grown as a tumor in BALB/c mice to obtain antibody-enriched ascites fluids. The ascites fluids were stored frozen at $-80^{\circ} \mathrm{C}$, after they had been centrifuged to remove precipitates.

Purification of monoclonal antibodies. Monoclonal antibodies were purified from mouse ascites fluids using an Affi-Gel protein A monoclonal antibody purification system (Bio-Rad Laboratories).

Properties of monoclonal antibodies. Each mAb was isotyped by immunodiffusion ${ }^{13)}$ using several anti-mouse immunoglobulin sera; their pIs were found by isoelectric focusing (IEF). ${ }^{14)}$
Specificity of monoclonal antibodies. The affinity of the $\mathrm{mAbs}$ for the domains or RCM-OM was compared with that for OM by solid-phase competitive RIA using ${ }^{125} \mathrm{I}$ labeled OM as previously reported. ${ }^{5}$ )

Paratope specificity of monoclonal antibodies. To test whether the mAbs recognized different epitopes on OM or not, a sandwich assay and solid-phase competitive RIA were used. The second antibodies in both assay $(10 \mu \mathrm{g}$ of $m A b 32 A 8,41 B 3$ and $51 B 3$ ) were iodinated with $0.2 \mathrm{mCi}$ ${ }^{125}$ I using chloramine $\mathrm{T}$ as the oxidant. ${ }^{15 \text { ) }}$

Sandwich assay. The wells of flexible microtiter plates (Falcon) were filled with $100 \mu \mathrm{l}$ of a suitable concentration of one $\mathrm{mAb}$ in phosphate-buffered saline (PBS) for $2 \mathrm{hr}$. The plates were then rinsed three times with PBS containing $0.05 \%$ Tween-20 (PBS-Tween) and treated with $125 \mu \mathrm{l}$ of $1 \%$ bovine serum albumin (BSA) in PBS for $2 \mathrm{hr}$ to avoid non-specific binding. After washing with PBSTween, $100 \mu \mathrm{l}$ of $\mathrm{OM}$ or the domains at saturating concentration in PBS were allowed to bind to the coated $\mathrm{mAb}$. The plates were washed, and finally, $100 \mu \mathrm{l}$ of a second antibody labeled with ${ }^{125} \mathrm{I}$ in PBS containing $0.1 \%$ gelatin (Gel-PBS) was added and incubated for $2 \mathrm{hr}$. Finally, the plates were rinsed, and the radioactivity of the second antibody bound to each well was measured by a $\gamma$-counter (Aloka).

Solid-phase competitive radioimmunoassay. Plates were coated with $100 \mu \mathrm{l}$ of antigens $(100 \mu \mathrm{g} / \mathrm{ml})$ and treated with BSA as already described. Ninety $\mu \mathrm{l}$ of non-labeled $\mathrm{mAb}$ (competitor) serially diluted in Gel-PBS was added and mixed with $10 \mu$ l of ${ }^{125} \mathrm{I}-\mathrm{mAb}$, before being incubated for $2 \mathrm{hr}$. The plates were rinsed with PBS-Tween, and the radioactivity of each well was measured. Relative values of the bound counts $(B / B o)$ were calculated with the equation: $B / B o=B^{\prime}-\mathrm{BKG} / B o^{\prime}-\mathrm{BKG}$ where $B^{\prime}$ is the ${ }^{125} \mathrm{I}-\mathrm{mAb}$ (cpm) bound to coated antigens in the presence of a competitor; $B o^{\prime}$ is the ${ }^{125} \mathrm{I}-\mathrm{mAb}(\mathrm{cpm})$ bound to coated antigens in the absence of a competitor; and $\mathrm{BKG}$ is the ${ }^{125} \mathrm{I}-\mathrm{mAb}(\mathrm{cpm})$ bound in the absence of coated antigens.

\section{RESULTS}

\section{Characterization of monoclonal antibodies}

Two monoclonal antibodies ( $m A b 23 E 5$ and $m A b 32 A 8)$ directed against OM had already been prepared, purified, and characterized as previously reported. ${ }^{5)}$ To prepare new antiOM mAbs different from these, lymphocytes from the OM-immunized BALB/c mice were hybridized, and some stable clones with specificity for OM were found. Two clones (41B3 and $51 B 3$ ) were selected for further characteri- 
TABle I. Properties of $m A b 41 B 3$ AND $m A b 51 B 3$

Each mAb was isotyped by immunodiffusion using various anti-mouse immunoglobulin sera, the pIs being measured by isoelectric focusing.

\begin{tabular}{lcc}
\hline $\mathrm{mAb}$ & $41 \mathrm{B3}$ & $51 \mathrm{B3}$ \\
\hline Class & $\mathrm{IgG}_{1}$ & $\mathrm{IgG}_{1}$ \\
Type & $\kappa$ & $\kappa$ \\
$\mathrm{pI}$ & 7.1 & 5.8 \\
\hline
\end{tabular}

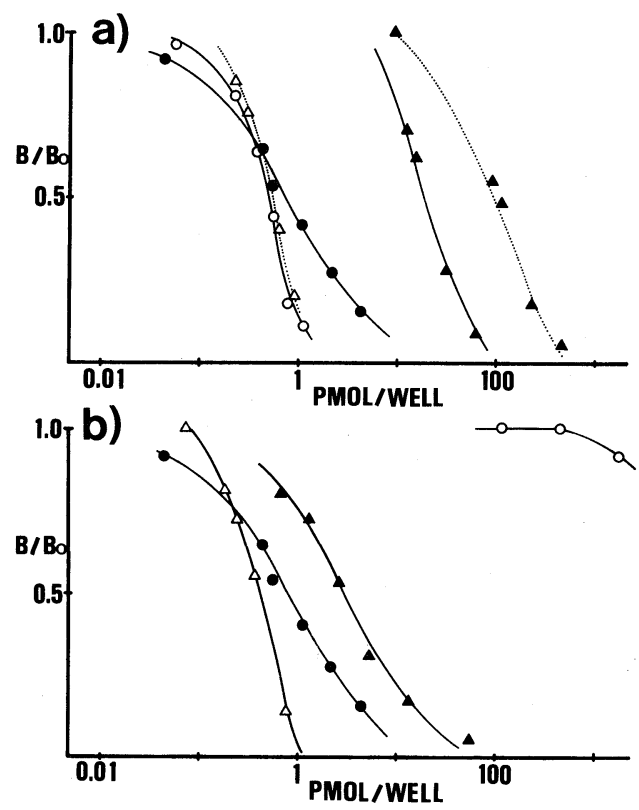

FIG. 1. Binding Curves of ${ }^{125} \mathrm{I}-\mathrm{OM}$ to $m A b 41 B 3$ in the Presence of Domains.

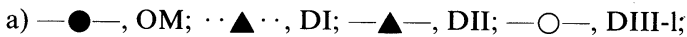
$\cdots \Delta \cdots$, DIII-2. b) $\bigcirc$, OM; $\boldsymbol{\Delta}$, DI-II; $\triangle$, DII-III; $O$, RCM-OM. $B / B o=$ bond ${ }^{125} \mathrm{I}-\mathrm{OM}$ to $\mathrm{mAb}$ in the presence of unlabeled antigens (competitors)/bound ${ }^{125} \mathrm{I}-\mathrm{OM}$ to $\mathrm{mAb}$ in the absence of competitors.

zation. Table I summarizes the properties of $m A b 41 B 3$ (produced by clone $41 B 3$ ) and $m A b$ $51 B 3$ (produced by clone 51B3). Isotypic analysis showed that both mAbs belonged to the $\mathrm{IgG}_{1}$ subclass with a $\kappa$ chain. It was shown by IEF that the $\mathrm{pI}$ of $m A b 41 B 3$ was 7.1 , and that of $m A b 51 B 3$ was 5.8 .

\section{Specificity of monoclonal antibodies}

The specificity of both mAbs was studied by solid-phase competitive RIA using various

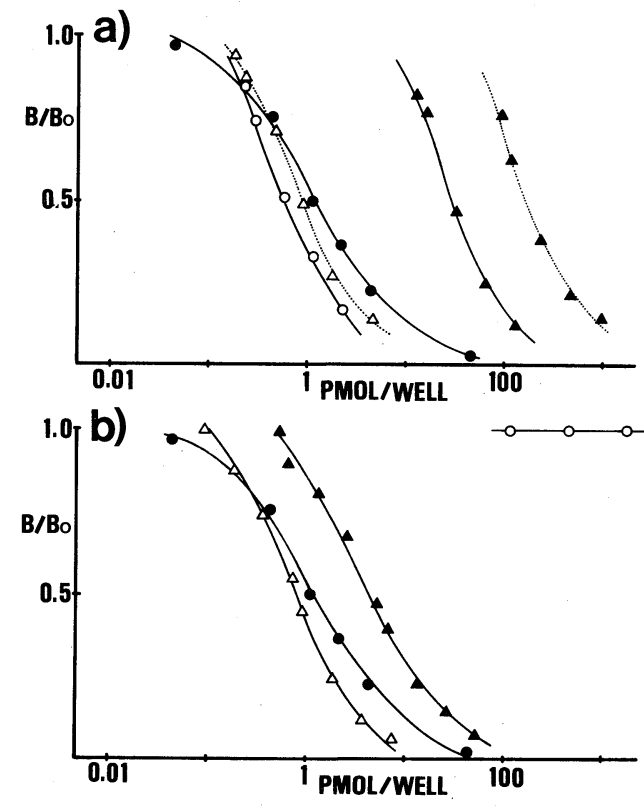

FIG. 2. Binding Curves of ${ }^{125} \mathrm{I}-\mathrm{OM}$ to $m A b 51 B 3$ in the Presence of Domains.

a) - - - OM; $\cdots \mathbf{\Delta} \cdots, \mathrm{DI} ;-\mathbf{\Delta}-$, DII; - O-, DIII-1; $\cdots \Delta \cdots$, DIII-2. b) O, OM; $\boldsymbol{\Delta}$, DI-II; $\triangle$, DII-III; $\bigcirc$, RCM-OM. $B / B o=$ bound ${ }^{125} \mathrm{I}-\mathrm{OM}$ to $\mathrm{mAb}$ in the presence of unlabeled antigens (competitors)/bound ${ }^{125} \mathrm{I}-\mathrm{OM}$ to $\mathrm{mAb}$ in the absence of competitors.

concentrations of $\mathrm{OM}$ and the domains to inhibit the binding of ${ }^{125} \mathrm{I}$-labeled OM to each $\mathrm{mAb}$ (Figs. 1 and 2). An increasing concentration of each unlabeled domain resulted in initially decreased binding, and finally fully inhibited the binding of ${ }^{125} \mathrm{I}-\mathrm{OM}$ to each $\mathrm{mAb}$. Thus, both mAbs could react with every domain of OM, but the affinities of the antibodies for the domains were not equal. Table II shows the values of $I_{50}$ calculated from the ratio of the concentration of each domain needed to inhibit $50 \%$ of the binding to ${ }^{125} \mathrm{I}-\mathrm{OM}$ to the concentration of unlabeled $\mathrm{OM}$ required to give the same inhibition. Lower values of $I_{50}$ mean higher affinities of $\mathrm{mAb}$ for the domain. The values of $\mathrm{I}_{50}$ for three mAbs $(23 E 5,41 \mathrm{B3}$, and $51 \mathrm{B3}$ ) resembled each other and, particularly, $\mathrm{I}_{50}$ of $m A b 41 B 3$ for each domain was very similar to that of $m A b 51 B 3$. Either $m A b$ $41 B 3$ or $m A b 51 B 3$ bound to fragments containing DIII (DIII-1 (free from carbohy- 
TABle II. $I_{50}$ OF THE Domains

The values of $I_{50}$ were calculated from the ratio of the molar concentration of each domain needed to inhibit $50 \%$ of the binding to ${ }^{125} \mathrm{I}-\mathrm{OM}$ to the molar concentration of unlabeled $\mathrm{OM}$ required to give $50 \%$ inhibition of the binding to ${ }^{125} \mathrm{I}-\mathrm{OM}$. The $\mathrm{I}_{50}$ values of $m A b$ $23 E 5$ and $m A b 32 A 8$ are cited from our previous report:

\begin{tabular}{lcccc}
\hline $\mathrm{mAb}$ & $23 E 5$ & $32 A 8$ & $41 B 3$ & $51 B 3$ \\
\hline OM & 1 & 1 & 1 & 1 \\
DI & 25.0 & 813 & 139 & 130 \\
DII & 14.7 & 39.8 & 24.7 & 25.9 \\
DIII-1 & 0.28 & 2140 & 0.49 & 0.70 \\
DIII-2 & 1.00 & 9.33 & 0.75 & 0.75 \\
DI-II & 24.2 & 1.07 & 3.80 & 3.86 \\
DII-III & 0.59 & 78.6 & 0.70 & 0.60 \\
\hline
\end{tabular}

drates), DIII-2 (with carbohydrates), and DIIIII) more efficiently than to fragments without DIII (DI, DII, and DI-II). These results raise the possibility that both $m A b 41 B 3$ and $m A b$ $51 B 3$ may mainly be directed against the polypeptide chain of DIII, and that these two $\mathrm{mAbs}$ recognize the same antigenic region. Neither $m A b 41 B 3$ nor $m A b 51 B 3$ could bind much to RCM-OM (Figs. 1 and 2) and it was found that they recognized the conformation of the antigen as well as did $m A b 23 E 5$ and $m A b 32 A 8$.

Association constants of monoclonal antibodies

The association constants $(K a)$ for the two $\mathrm{mAbs}$ for $\mathrm{OM}$ were found from Scatchard plots (Fig. 3); the $K a$ for $m A b 41 B 3$ was $1.26 \times$ $10^{8} \mathrm{l} / \mathrm{mol}$ and for $m A b 51 B 3,2.29 \times 10^{8} 1 / \mathrm{mol}$. These values are higher than that for $m A b$ $23 E 5\left(K a=2.20 \times 10^{7} 1 / \mathrm{mol}\right)$. The $K a$ for $m A b$ $32 A 8$ could not be calculated as the Scatchard plot did not give a straight line.

\section{Sandwich assay}

To find whether the four mAbs $(23 E 5,32 A 8$, $41 B 3$, and $51 B 3$ ) recognized the same epitope on the ovomucoid molecule, a sandwich assay and solid-phase competitive RIA were done. In the sandwich assay, one of the four mAbs was fixed in the wells at first. When exposed to antigens (OM, DI, DII, DIII-1, DIII-2, DI-II, or DII-III), the coated $\mathrm{mAb}$ would bind to

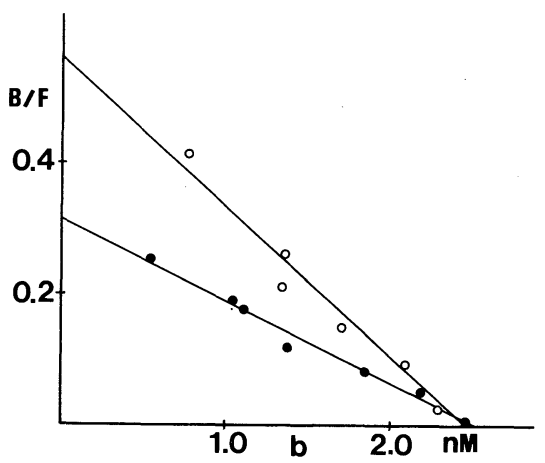

FIG. 3. Scatchard Plots of Two Monoclonal Antibodies against OM.

, $m A b 41 B 3 \quad Y=-1.26 \times 10^{8} X+0.316 ; \bigcirc, m A b 51 B 3$ $Y=-2.29 \times 10^{8} X+0.561 ; \quad B / F=$ bound $/$ free $\quad{ }^{125} \mathrm{I}-\mathrm{OM}$; $b=$ amount of bound OM.

restricted regions on the antigen molecule. Then, a ${ }^{125}$ I-labeled $\mathrm{mAb}$ (the second $\mathrm{mAb}$ ) was added, and this would only bind to the antigen molecules that were fixed on the solid phase (bound to the coating $\mathrm{mAb}$ ) and had the epitope specific for the second $\mathrm{mAb}$ but different from the coating $\mathrm{mAb}$. Thus, the radioactivity remaining in each well allowed detection of the simultaneous binding of two antibodies on the antigen molecules. Low radioactivity would generally mean that the antibodies did not simultaneously bind because the epitopes on the antigen molecule directed against two antibodies were the same or near. On the other hand, high radioactivity would indicate that the antibodies could simultaneously bind because the epitopes directed against the antibodies were distinct. Table III demonstrates the results of the sandwich assay using ${ }^{125} \mathrm{I}$-labeled $m A b 32 A 8,41 \mathrm{B3}$, and $51 \mathrm{B3}$. When the solid phase antibody and the second antibody were the same, or the two antibodies were $m A b 41 B 3$ and $m A b 51 B 3$, or $m A b 32 A 8$ was used as the solid phase or the second antibody in presence of DIII (DIII-1 or DIII2 ), the radioactivity was low.

\section{Solid-phase competitive radioimmunoassay}

The results of a competitive analysis of the four anti-OM mAb by RIA using ${ }^{125}$ I-labeled $\mathrm{mAbs}(32 A 8,41 B 3$, and $51 B 3)$ are presented in 
Table III. Sandwich Assay of Four MONOClONAL ANTIBODIES

Plates coated with one of the four mAbs (solid phase $\mathrm{mAb}$ ) were incubated with each domain. (a), OM; b), DI; c), DII; d), DIII-1; e), DIII-2; f), DI-II; g), DII-III) Then, a ${ }^{125}$ I-labeled $\mathrm{mAb}$ (one of $m A b 32 A 8, m A b 41 B 3$ and $m A b 51 B 3$ ) was added and incubated. The readioactivity remaining in each well was measured.

\begin{tabular}{crrrr}
\hline & \multicolumn{4}{c}{ Solid phase $\mathrm{Ab}$} \\
\cline { 2 - 5 } a) & $23 E 5$ & $32 A 8$ & $41 B 3$ & $51 B 3$ \\
\hline Labeled Ab & & & & \\
$32 A 8$ & 2971 & 9 & 10967 & 10188 \\
$41 B 3$ & 8427 & 9871 & 130 & 27 \\
$51 B 3$ & 11247 & 8892 & 16 & 0 \\
\hline
\end{tabular}

b)

Solid phase $\mathrm{Ab}$

\begin{tabular}{crrrr}
\cline { 3 - 4 } b) & $23 E 5$ & $32 A 8$ & $41 B 3$ & $51 B 3$ \\
\hline Labeled Ab & & & & \\
$32 A 8$ & 4020 & 2 & 6720 & 8895 \\
$41 B 3$ & 8899 & 7093 & 45 & 37 \\
$51 B 3$ & 14081 & 9673 & 9 & 0 \\
\hline
\end{tabular}

\begin{tabular}{crrrr}
\hline & \multicolumn{4}{c}{ Solid phase Ab } \\
\cline { 2 - 5 } c) & $23 E 5$ & $32 A 8$ & $41 B 3$ & $51 B 3$ \\
\hline Labeled Ab & & & & \\
$32 A 8$ & 4779 & 9 & 7235 & 7224 \\
$41 B 3$ & 9037 & 4986 & 18 & 35 \\
$51 B 3$ & 12891 & 7399 & 11 & 39 \\
\hline
\end{tabular}

d)

Solid phase $\mathrm{Ab}$

\begin{tabular}{crrrr} 
d) & $23 E 5$ & $32 A 8$ & $41 B 3$ & $51 B 3$ \\
\cline { 2 - 5 } Labeled Ab & & & & \\
$32 A 8$ & 0 & 4 & 8 & 0 \\
$41 B 3$ & 9029 & 58 & 0 & 80 \\
$51 B 3$ & 13163 & 0 & 12 & 32 \\
\hline
\end{tabular}

e)

Solid phase $\mathrm{Ab}$

\begin{tabular}{crrrr}
\cline { 3 - 5 } e) & $23 E 5$ & $32 A 8$ & $41 B 3$ & $51 B 3$ \\
\hline Labeled Ab & & & & \\
$32 A 8$ & 0 & 0 & 0 & 0 \\
$41 B 3$ & 10434 & 115 & 53 & 52 \\
$51 B 3$ & 13847 & 20 & 6 & 0 \\
\hline
\end{tabular}

\begin{tabular}{|c|c|c|c|c|}
\hline \multirow{2}{*}{ f) } & \multicolumn{4}{|c|}{ Solid phase $\mathrm{Ab}$} \\
\hline & $23 E 5$ & $32 A 8$ & $41 B 3$ & $51 B 3$ \\
\hline \multicolumn{5}{|c|}{ Labeled $\mathrm{Ab}$} \\
\hline $32 A 8$ & 2101 & 0 & 10122 & 6851 \\
\hline $41 B 3$ & 11958 & 9611 & 51 & 27 \\
\hline \multirow[t]{2}{*}{$51 B 3$} & 11684 & 4263 & 0 & 0 \\
\hline & \multicolumn{4}{|c|}{ Solid phase $\mathrm{Ab}$} \\
\hline g) & $23 E 5$ & $32 A 8$ & $41 B 3$ & $51 B 3$ \\
\hline \multicolumn{5}{|c|}{ Labeled Ab } \\
\hline $32 A 8$ & 5100 & 0 & 4392 & 4902 \\
\hline $41 B 3$ & 9045 & 8842 & 297 & 52 \\
\hline $51 B 3$ & 14264 & 12612 & 26 & 0 \\
\hline
\end{tabular}

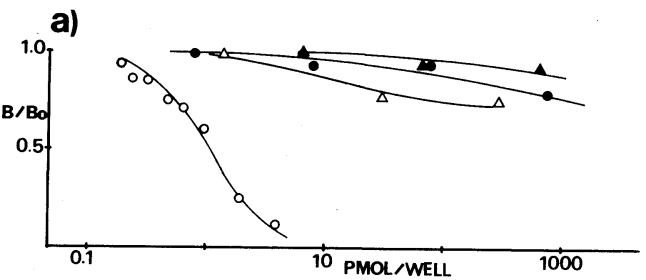

b)
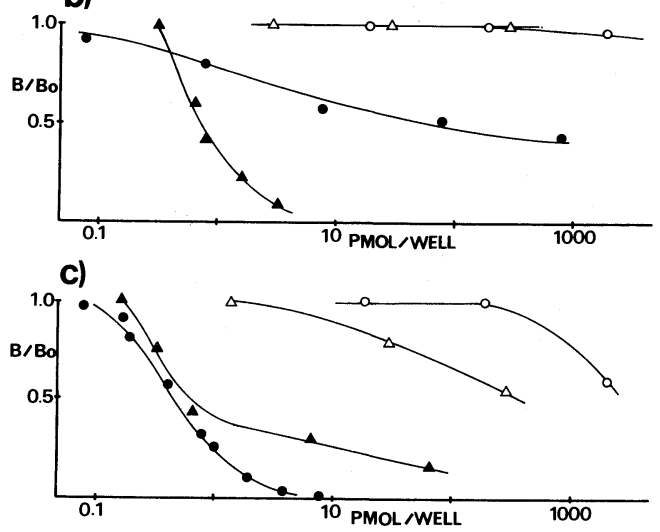

FIG. 4. Solid-phase Radioimmunoassay of the Paratope Specificity of Anti-OM Monoclonal Antibodies.

OM coated plates were incubated with serial dilutions of each $\mathrm{mAb}(\triangle, m A b 23 E 5 ; O, m A b 32 A 8 ; \mathbf{\Delta}, m A b 41 B 3$; - $m A b 51 B 3)$ and constant amounts of ${ }^{125} \mathrm{I}-\mathrm{mAb}(\mathrm{a})$, $m A b 32 A 8$; b), $m A b 41 B 3$; c), $m A b 51 B 3)$. When 1) the labeled and coated mAbs were the same and 2) the two antibodies were $m A b 41 B 3$ and $m A b 51 B 3$, higher inhibitions were shown. 
a)

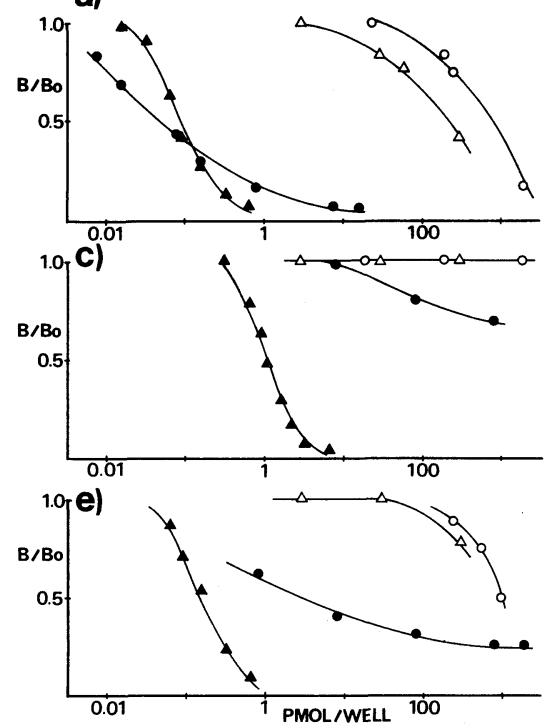

b)
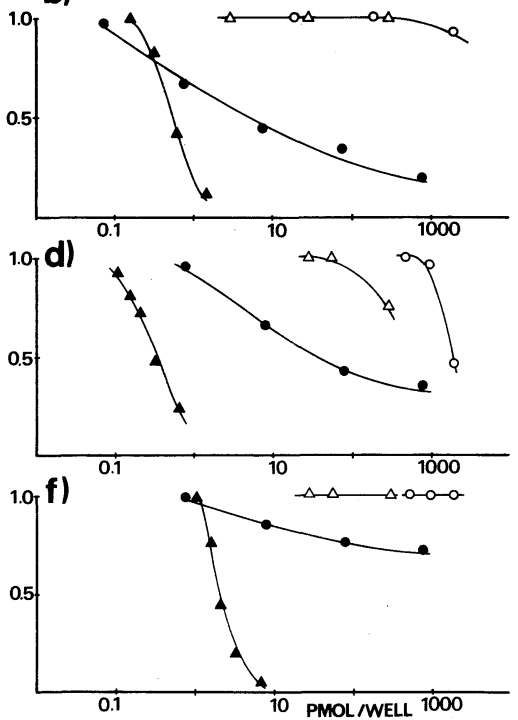

FIG. 5. Solid-phase Radioimmunoassay of the Paratope Specificity of Anti-OM Monoclonal Antibodies.

Plates coated with one of the domains ( a), DI; b), DII; c), DIII-l; d), DIII-2; e), DI-II; f), DII-III) were incubated with serial dilutions of each $\mathrm{mAb}(\triangle, m A b 23 E 5 ; \bigcirc, m A b 32 A 8 ; \mathbf{\Delta}, m A b 41 B 3 ; \boldsymbol{O}, m A b 51 B 3)$ and constant amount of ${ }^{125} \mathrm{I}-m A b 41 B 3$. Both $m A b 41 B 3$ and $m A b 51 B 3$ inhibited the binding of ${ }^{125} \mathrm{I}-m A b 41 B 3$ to antigens.

a)
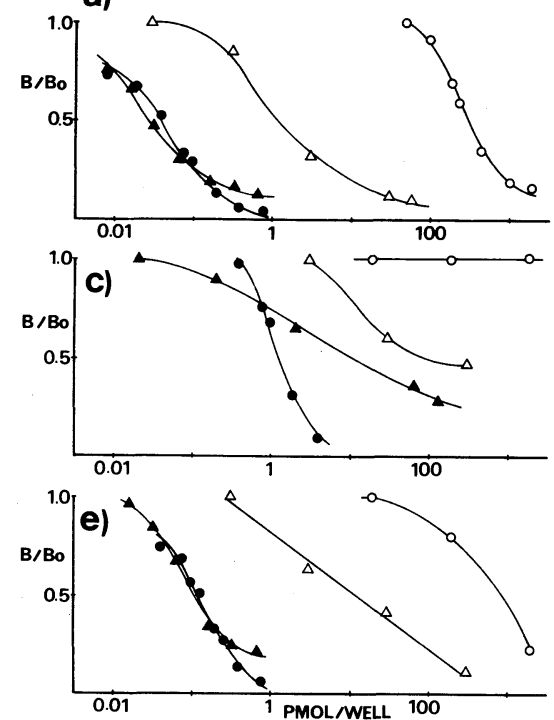

b)
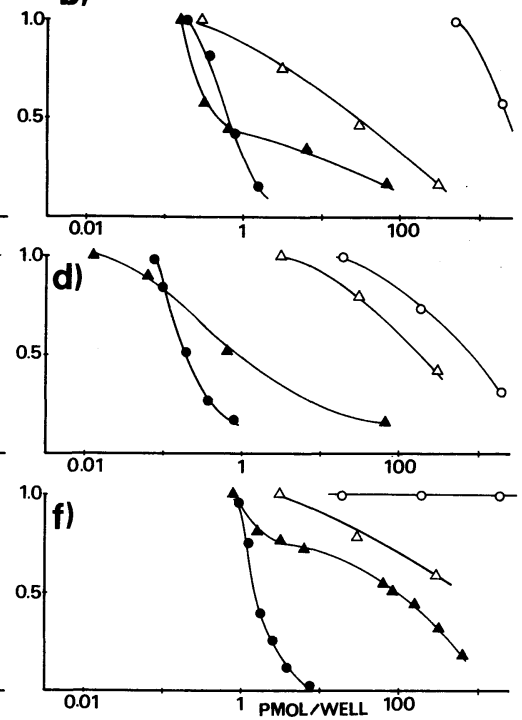

FIG. 6. Solid-phase Radioimmunoassay of the Paratope Specificity of Anti-OM Monoclonal Antibodies.

Plates coated with one of the domains ( a), DI; b), DII; c), DIII-1; d), DIII-2; e), DI-II; f), DII-III) were incubated with serial dilutions of each $m A b(\triangle, m A b 23 E 5 ; \bigcirc, m A b 32 A 8 ; \boldsymbol{\Lambda}, m A b 41 B 3 ; \boldsymbol{\bullet}, m A b 51 B 3)$ and constant amount of ${ }^{125} \mathrm{I}-m A b 51 B 3$. Both $m A b 41 B 3$ and $m A b 51 B 3$ inhibited the binding of ${ }^{125} \mathrm{I}-m A b 51 B 3$ to antigens. 
Figs. 4, 5, and 6. When the labeled and unlabeled $\mathrm{mAbs}$ were the same, increasing the quantity of a competitor allowed $100 \%$ of the binding activity of labeled $\mathrm{mAb}$ to the antigens to be inhibited. When they were not the same, various degrees of inhibition were found. Both $m A b 41 B 3$ and $m A b 51 B 3$ caused high inhibition, but little inhibition was shown by other pairs. It is generally concluded that a high inhibition means the same or resembling paratope specificities of two mAbs, and that a low inhibition means distinct paratope specificity. These results are consistent with those of the sandwich assay.

\section{DISCUSSION}

The antigenic structures of OM have already been studied using rabbit antiserum and mouse mAb (23E5 and 32A8) directed against OM. ${ }^{5,16 \sim 19)}$ Ovomucoid is a glycoprotein with heterogenous carbohydrates and consists of three homologous domains that each have three intramolecular disulfide bonds. The epitope structures of $\mathrm{OM}$ are closely related to these features. From our previous work, we concluded that 1) one antibody specific for OM can bind all three domains, although the affinities are not. equal, that is, each domain has common epitopes because of their homology; 2) almost all the anti-OM antibodies recognize the conformation of antigens; 3 ) the specific anti-OM antibodies have generally the highest association constant for DIII, then DII > DI; and 4) the sugar chains are important in binding OM to some of the antibodies, that is, it is likely that some of them recognize the region on OM containing the carbohydrates.

For this paper, we prepared, purified, and characterized two mAbs (41B3 and 51B3) different from those previously reported. Both $m A b 41 B 3$ and $m A b 51 B 3$ reacted most efficiently with DIII in the three domains and also had slightly more affinity for DIII-1 (free from carbohydrates) than for DIII-2 (with carbohydrates). These results suggest that they mainly recognize a region on the polypeptide chain of DIII.

From Scatchard plots we calculated the $K a$ of each $\mathrm{mAb}$ for $\mathrm{OM}$, with $K a$ values for $m A b$ $23 E 5,41 B 3$, and $51 B 3$ of $2.20 \times 10^{7}, 1.26 \times 10^{8}$, and $2.29 \times 10^{8} \mathrm{l} / \mathrm{mol}$, respectively. These three $\mathrm{mAbs}$ were similar in their binding to $\mathrm{OM}$ and the domains, but not identical. The Scatchard plot for $m A b 32 A 8$ did not give a straight line, probably because the sugar chains of $\mathrm{OM}$ were concerned in the binding of $m A b 32 A 8$ to $\mathrm{OM}$, which consisted of heterogenous carbohydrates.

To analyze the paratope specificity of the four $\mathrm{mAbs}$ directed against $\mathrm{OM}$, the sandwich assay and solid-phase competitive RIA were done. These two quite different assays gave similar results. Only the $\mathrm{mAb}$ pair of $41 \mathrm{B3}$ and $51 B 3$ could not simultaneously bind the antigens in the sandwich assay. Only $m A b 41 B 3$ inhibited the binding of $m A b 51 B 3$ to $\mathrm{OM}$ or the domains and vice versa in the solid-phase competitive RIA. However, these two assays did not give similar results when DIII was used as the antigen. An excess of $m A b 32 A 8$ did not inhibit the binding of DIII to $m A b 41 B 3$ or $m A b 51 B 3$ in the solid-phase competitive RIA. These results indicate that $m A b 41 B 3$ and $m A b$ $51 B 3$ recognized regions distinct from the binding site to $m A b 32 A 8$, although the sandwich assay showed that the mAb pair of $32 A 8$ and $41 B 3$, or that of $m A b 32 A 8$ and $m A b 51 B 3$, did not simultaneously bind DIII. According to the analysis of paratope specificity, the four $m A b s$ can be classified into three groups that are directed against distinct epitopes of OM; that is, $m A b 41 B 3$ and $m A b 51 B 3$ belong to the same group distinct from $m A b 23 E 5$ or $m A b$ $32 A 8$. However, it is not clear by these two assays whetehr $m A b \quad 41 B 3$ and $m A b 51 B 3$ recognized exactly the same antigenic site, overlapping sites, two close sites, or a conformational-dependent distinct site. Ovomucoid consists of three homologous domains, and each domain has common epitopes. Therefore, it is possible that two of the same mAbs could bind to one ovomucoid molecule simultaneously, but this was not shown by these assays. 
Protein antigens generally have several antigenic sites on one molecule. At present, it is not clear whether one antigenic site is correlated to another antigenic site on the same molecule or not. For example, studies using $\mathrm{mAb}$ against distinct regions of the protein antigens insulin ${ }^{20)}$ and myoglobulin ${ }^{21)}$ showed that an anti-idiotypic antiserum to one $\mathrm{mAb}$ inhibited antigen binding by another mAb. Thus, these mAbs binding to distinct sites of one protein antigen have been found to express shared idiotypes. These results suggest that these epitopes on one antigen are related to each other. A mAb specific for horse cytochrome $c,{ }^{22)}$ however, did not express shared idiotypes. It is said from this result that these epitopes are completely distinct as well as those of other protein antigens in their immune response.

We have now prepared four mAbs specific for $O M$ that recognize at least three distinct epitopes of OM as just described. These mAbs may be useful for general studies on epitopes of protein antigens as well as for analyses of the antigenic determinants of OM.

\section{REFERENCES}

1) B. Gjesing and H. Løwenstein, Ann. Allergy, 531, 602 (1984).

2) E. Bleumink and E. Young, Int. Arch. Allergy, 40, 72 (1971).

3). I. Kato, Tanpakushitsu Kakusan-Kouso, 24, 667 (1979).
4) K. Yamashita, J. P. Kamerling and A. Kobata, J. Biol. Chem., 258, 3099 (1983).

5) S. Kaminogawa, A. Enomoto, J. Kurisaki and K. Yamauchi, J. Biochem., 98, 1027 (1985).

6) E. Fredericq and H. F. Deutsch, J. Biol. Chem., 181, 499 (1949)

7) I. Kato, W. J. Kohr and M. Laskowski, Jr., Fed. Proc., 36, 764 (1978).

8) J. G. Beeley, Biochem. J., 155, 345 (1976).

9) J. G. Beeley and E. Mccairns, Biochim. Biophys. Acta, 271, 204 (1972).

10) W. Konigsberg, "Methods in Enzymology," Vol. 25, ed. by C. H. W. Hirs and S. N. Timasheff, Academic Press, NewYork, 1972, p. 185.

11) G. Köhler and C. Milstein, Nature, 256, 495 (1975).

12) E. Engvall and P. Perlmann, Immunochemistry, 8, 871 (1971).

13) Ö. Ouchterlony and L. Å. Nilsson, "Handbook of Experimental Immunology," Vol. 1, ed. by D. M. Weir, Blackwell Scientific Publications, 1973, Chapter 19.

14) Z. L. Awdeh, A. R. Williamson and B. A. Askonas, Nature, 219, 66 (1968).

15) F. C. Greenwood and W. M. Hunter, Biochem. J., 89, 114 (1963).

16) Y. Konishi, J. Kurisaki, K. Yamauchi and S. Kaminogawa, Jpn. J. Zootech. Sci., 52, 542 (1981).

17) J. Kurisaki, Y. Konishi, S. Kaminogawa and K. Yamauchi, Agric. Biol. Chem., 45, 879 (1981).

18) Y. Konishi, J. Kurisaki, S. Kaminogawa and K. Yamauchi, Agric. Biol. Chem., 46, 305 (1982).

19) Y. Konishi, J. Kurisaki, S. Kaminogawa and K. Yamauchi, J. Food Sci., 50, 1422 (1985).

20) T. P. Bender, J. Schroer and J. L. Claflin, J. Immunol., 131, 2882 (1983).

21) Y. Kohno, I. Berkower, J. Minna and J. A. Berzofsky, J. Immunol., 128, 1742 (1982).

22) F. R. Carbone and Y. Paterson, J. Immunol., 135, 2609 (1985) 